2010-01-01

\title{
Experimental Analysis and Demonstration of a Low Cost Fibre Optic Temperature Sensor System for Engineering Applications
}

\author{
Ginu Rajan \\ Technological University Dublin, ginu.rajan@tudublin.ie \\ Yuliya Semenova \\ Technological University Dublin, yuliya.semenova@tudublin.ie \\ Jinesh Mathew \\ Technological University Dublin
}

See next page for additional authors

Follow this and additional works at: https://arrow.tudublin.ie/engscheceart

Part of the Engineering Commons

\section{Recommended Citation}

Rajan, G. et al. (2010) Experimental analysis and demonstration of a low cost fibre optic temperature sensor system for engineering applications, Sensors \& Actuators: A. Physical, No. 163, pp 88-95. doi:10.1016/j.sna.2010.07.010

This Article is brought to you for free and open access by the School of Electrical and Electronic Engineering at ARROW@TU Dublin. It has been accepted for inclusion in Articles by an authorized administrator of ARROW@TU Dublin. For more information, please contact arrow.admin@tudublin.ie, aisling.coyne@tudublin.ie, gerard.connolly@tudublin.ie.

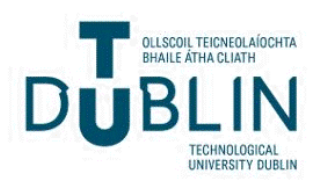




\section{Authors}

Ginu Rajan, Yuliya Semenova, Jinesh Mathew, and Gerald Farrell

This article is available at ARROW@TU Dublin: https://arrow.tudublin.ie/engscheceart/93 


\title{
Experimental analysis and demonstration of a low cost fibre optic temperature sensor system for engineering applications
}

\author{
Ginu Rajan, Yuliya Semenova, Jinesh Mathew and Gerald Farrell \\ Photonics Research Centre, School of Electronic and Communications Engineering, \\ Dublin Institute of Technology, Dublin 8, Ireland \\ E-mail: ginu.rajan@dit.ie
}

\begin{abstract}
An epoxy packaged surface mountable fibre temperature sensor for engineering applications is presented in this paper. The temperature sensor is based on a macro-bend single-mode fibre loop employed in a ratiometric power measurement scheme and has a linear characteristic with temperature at a fixed wavelength and bend radius. The sensor head consists of a single turn of a bare bend sensitive single-mode fibre with an applied absorption coating. The temperature of the sensor head is varied up to $80{ }^{0} \mathrm{C}$ and the linearity of the response is studied with different applied absorption coatings. The sensor head is packaged in a low thermal expansion coefficient two part epoxy for stability. Such a packaged sensor head can be directly attached to any structure for temperature measurements. The packaged sensor system shows a temperature resolution better than $0.5{ }^{0} \mathrm{C}$. The impact of stress and strain on the fabricated sensor is investigated by applying an external load to the sensor and an estimation of magnitude of the stress and strain induced error in the temperature measurement of the system has been obtained. It is found that the impact of stress and strain is low and can be compensated, if necessary. A portable prototype of the sensor head and the interrogation system is also demonstrated in this paper. The demonstrated temperature sensing system could be used for a wide range of engineering applications.
\end{abstract}




\section{Introduction}

The bend loss properties of single-mode fibre have been studied very intensively $[1,2]$ along with applications such as a fibre filter based on the bend loss phenomenon utilized as an edge filter for wavelength measurements [3]. A significant amount of effort has been expended by researchers on the use of the bend loss properties of single-mode fibre for temperature measurements. One method is based on interferometry [4] where the temperature sensitivity arises from the thermo-optic and thermal expansion sensitivity of the buffer coating leading to interference between the whispering gallery (WG) modes and the core mode. This method requires the determination of phase information at different wavelengths to extract the temperature information. High temperature sensing using WG mode resonance in bent fibres is also reported [5], which involves measuring the resonance wavelength peak shift with temperature. Both approaches lack a direct linear relation between bend loss and temperature and the need for phase measurements makes the system complex.

Another common and widely used fibre sensor for temperature and strain measurements are the fibre Bragg grating (FBG) sensors [6, 7]. In the case of fibre Bragg gratings, the temperature resolution of the sensor depends on the type of interrogation technique used to covert the wavelength shift information into temperature information. Highly expensive interferometric techniques are required to resolve sub- degree temperature and this makes the entire system less cost effective [8]. The improvements in the fabrication of FBGs have reduced the cost of grating fabrication, so that the interrogation unit, rather than the sensor, accounts for a large proportion of the cost of a complete sensing system. The work presented in this paper describes the development of a complete sensor system consisting of the sensor head and the interrogation system, which is cost effective and simple in nature when compared to other fibre sensor systems.

We have recently demonstrated the possibility of using a macro-bend fibre as a temperature sensor [9]. A bend sensitive buffer stripped single mode fibre with a suitable absorption coating is employed in a ratiometric scheme for temperature measurements. The removal of the buffer coating eliminates the effect of the two different thermo- optic 
coefficients (TOC) of the buffer and the cladding and the use of an appropriate absorption layer results in a monotonic increase in bend loss with a decrease in the bending radius which is equivalent to the behaviour of a core-infinite cladding structure [10]. This paper is focussed on a detailed analysis of the impact of the coating on the temperature response and on the utilization of the macro-bend based temperature sensor for engineering applications. The sensor head is packaged in a low thermal expansion coefficient two-part epoxy for mechanical stability. The developed temperature sensor can be directly attached to any structure for temperature measurements. A complete calibration of the sensor system taking account of the temperature response of the packaged sensor, temperature resolution and the stress and strain induced temperature errors are presented in this paper. In section 3, the impact of the absorption coating on the temperature response is presented, while in section 4 a performance evaluation of the packaged sensor is discussed. A portable prototype of the sensor system is also demonstrated and is presented in section 5 of this paper.

\section{Background}

The temperature sensitive sensor head consists of a buffer stripped bend-sensitive singlemode fibre arranged in a loop with an absorption layer applied to the cladding as shown in Fig. 1(a). The absorption layer should be chosen to absorb light at the wavelength of operation, suppressing the WG modes inherent in a bent single-mode fibre and reducing the reflections back from the air-cladding boundary. By eliminating the WG modes, the bend loss variation with temperature at a fixed wavelength and loop bend radius depends only on the thermo-optic coefficients of the cladding and core [11].

By removing the buffer coating from the sensor head, the only negative TOC material is removed and the sensor head consists of only positive TOC materials; the cladding and core, which are made of silica material. For the silica core and cladding the thermally induced effective change in refractive index is linear in nature, resulting in a linear variation of bend loss with temperature. Furthermore, there is a monotonic increase in bend loss with a decrease in the bend radius and with an increase in the wavelength [11]. 
Thus the temperature sensitivity of the sensor can be varied by changing the bend radius or the operating wavelength.

The temperature information is extracted using a simple ratiometric power measurement system which measures a ratio which is independent of source power variations resulting in a more stable and accurate system. The input signal from the source splits into two equal power signals, one goes to the fibre sensor and the other one is the reference signal as shown in Fig. 1(b). Two photodiodes plus associated electronics are used to measure the power at the outputs of the corresponding arms. By measuring the power ratio of the two signals, which is a function of temperature, temperature can be measured, assuming the system is properly calibrated.

\section{Temperature response of the sensor}

The fibre used in the experimental investigation was a 1060XP single-mode fibre which has a high bend loss in the wavelength region circa $1550 \mathrm{~nm}$ and gives an excellent bend loss response with wavelength as we reported earlier [12]. To fabricate the macro-bend fibre temperature sensor the buffer coating is stripped from the middle section of a length of the fibre and an absorption layer is applied to the stripped section. The absorption coatings used in this investigation are readily available coatings such as permanent marker ink, Indian ink and black matt paint. The absorption properties of the materials are firstly studied at room temperature. The coating materials are coated on a glass plate (micro slide) and absorption spectra of the materials are measured using an absorption spectrometer (Perkin Elmer Lambda 900 UV/VIS/NIR Spectrometer) for a wavelength range of $400-1700 \mathrm{~nm}$ with an interval of $1 \mathrm{~nm}$. The measured transmission spectra of the coating materials are shown in Fig 2(a). From the Fig. 2(a) it is clear that all the materials have a good absorption at the visible wavelength range. However only the Indian ink shows an approximately 100\% absorption of light in the wavelength range of $1500-1600 \mathrm{~nm}$. The reflected spectrum of the Indian ink is also measured and we found no reflected signal. It is therefore reasonable to conclude that $100 \%$ absorption is taking place. For a further insight the 0-5\% transmission values of the coatings are shown in Fig. 2(b). The black matt paint absorbs nearly 96\% while the permanent marker ink's 
absorption is very poor in the IR range making both materials unsuitable as an absorption coating for the macro-bend fibre temperature sensor.

In order to analyze and compare the impact of the absorption coating on the temperature response, three bare macro-bend fibre devices were fabricated. Three samples of 1060XP fibres were buffer stripped and coated with one of the three different absorption materials: Indian ink, black matt paint and permanent marker ink respectively. The coatings were applied to the buffer stripped region of the fibre manually. The absorption properties of the coatings applied to the fibre may not be exactly the same for the glass plate samples; however, the overall trends remain the same. The sensor head is formed by creating a single fibre loop of $360^{\circ}$ by inserting the fibre ends into a short $2 \mathrm{~mm}$ polymer tube to support the overlap junction of the loop. In order to fix the radius, the junction of the fibre inside the tube is glued (using Loctite extreme). This forms a macro-bend fibre temperature sensor head. For the studies of the effect of coating the bend radius used was $12.5 \mathrm{~mm}$ which gives a bend loss of $7 \mathrm{~dB}$ at $0{ }^{0} \mathrm{C}$ for a wavelength of $1550 \mathrm{~nm}$. The sensor is utilized in the ratiometric power measurement scheme as shown in Fig 1(b). The input wavelength to the system was $1550 \mathrm{~nm}$. The fibre loop was fixed to a $5 \mathrm{~cm}$ diameter aluminium plate whose temperature was controlled using a Peltier cooler driven by a temperature controller. Full contact between the fibre sensor and the base plate was ensured. Using an accurate independent temperature monitor for the purpose of calibration, the ratio response was measured at $2{ }^{0} \mathrm{C}$ intervals for the temperature range of $0{ }^{0} \mathrm{C}$ to $+75{ }^{\circ} \mathrm{C}$. A 2 minute time interval was allowed between each measurement to ensure that the temperature of all parts of the sensor is uniform and stable. The range employed $\left(0{ }^{0} \mathrm{C}-75{ }^{0} \mathrm{C}\right)$ was limited by the capabilities of the Peltier cooler used. The ratio responses of all the fibre devices were measured individually. The measured ratio as a function of temperature is shown in Fig. 3(a).

From Fig. 3(a) it can be seen that the sensor head coated with Indian ink has a temperature response closest to a linear spectrum. Fig. 3(a) indicates that the radiation modes are not efficiently suppressed in bent fibres coated with black matt paint and the permanent marker ink and almost a complete suppression of the WG effect is required for 
the bend loss phenomenon to be used for temperature sensing. In the case of fibre coated with Indian ink, since the WG modes effect is eliminated completely, the macro-bend fibre gives a linear variation of bend loss with temperature and can be used as a temperature sensor. The temperature sensitivity of the sensor can be improved by using a fibre loop of smaller radius. In Fig. 3(b) a comparison of measured ratio versus temperature response of sensors with a bend radius of $11.5 \mathrm{~mm}$ and $12.5 \mathrm{~mm}$ is shown. It is clear from the figure that temperature sensitivity is substantially increased by reducing the bend radius. Thus based on the required temperature sensitivity different fibre loop radii can be used.

\section{Performance evaluation of the packaged sensor}

Since the sensor head is made of fragile single-mode fibre, suitable packaging is required for the sensor to be used in most applications. The sensor head selected for packaging is the one with $11.5 \mathrm{~mm}$ bend radius due to its high temperature sensitivity. The sections presented below explain the packaging method, temperature response and resolution of the packaged sensor. The effect of stress and strain on the packaged sensor is also discussed.

\subsection{Packaging of the sensor head}

For packaging of the sensor head a two part epoxy (353ND) with a low coefficient of thermal expansion is used. The thermal expansion coefficient of the epoxy below $120{ }^{0} \mathrm{C}$ (Transition temperature) is $54 \times 10^{-6} \mathrm{~K}^{-1}$. The Young's Modulus of the epoxy used was 6.2 GPa. The thermal conductivity of the epoxy was $0.34 \mathrm{~W} / \mathrm{mK}$ and it can withstand temperatures in the range of $-50{ }^{0} \mathrm{C}$ to $180{ }^{0} \mathrm{C}$. The curing time for the epoxy is $15-30$ minutes at $80{ }^{0} \mathrm{C}$.

Practically for packaging the sensor initially a circular aluminium tray was fixed with plastic tubes to support the macro-bent fibre temperature sensor as shown in Fig. 4(a). The Indian ink coated fibre was then fixed to the tray with a $360^{\circ}$ loop, with a bend radius of approximately $11.5 \mathrm{~mm}$, with the two ends spliced to standard singlemode fibre (smf28) pigtails. The two-part epoxy (353ND) is then mixed thoroughly and poured into 
the aluminium tray. Good care had been taken to make sure that the sensor stays almost in the middle part of the epoxy, to ensure a uniform distance from the top and bottom of the epoxy layer. The epoxy filled tray is then placed on a hot plate and a temperature of $80{ }^{0} \mathrm{C}$ is applied for a time interval of 30 minutes to cure the epoxy. Once the epoxy is cured to the solid state, the aluminium tray is removed carefully. This completes the packaging of the fibre temperature sensor. The average thickness of the packaged fibre sensor was $2.5 \mathrm{~mm}$. The sequence of the packaging steps is shown in Fig 4.

\subsection{Temperature response and resolution of the packaged sensor}

To study the temperature response of the packaged sensor, the experimental setup used was the same as that for the unpackaged sensor head. The packaged sensor is fixed to a Peltier cooler and the temperature is varied from $0{ }^{\circ} \mathrm{C}$ to $80{ }^{\circ} \mathrm{C}$, at an interval of $2{ }^{\circ} \mathrm{C}$. A 2 minute time interval is allowed in between each measurements, so that the Peltier cooler can attain temperature stability. The laser source used was a tunable laser Nettest 1560. The ratio vs. wavelength response of the packaged sensor is also obtained, and from Fig. 5(a) it can be seen that the ratio increases with wavelength monotonically. This indicates that at higher wavelengths, the bend loss is higher and since the temperature dependent loss of the bent fibre is proportional to the bend loss [11], the temperature sensitivity of the sensor will be higher at longer wavelengths. To underpin the ease of fabrication and to show the repeatability of the response of the sensor, a comparison of the ratio response of the sensor versus wavelength and temperature, before and after packaging is also presented in Fig. 5(a) and Fig. 5(b) respectively. From the figure it is clear that both the responses before and after packaging are very close and show very similar characteristics. The slight difference is due to the difference in the thermal expansion coefficient values of the silica material and the epoxy which could induce pressure on the sensor as the temperature increases. However this will not affect the accuracy of temperature measurement of the packaged sensor as the sensor is calibrated after packaging.

To study the wavelength dependent temperature sensitivity of the packaged sensor, the temperature response of the sensor is measured at three different wavelengths, $1530 \mathrm{~nm}$, $1550 \mathrm{~nm}$ and $1570 \mathrm{~nm}$ and is shown in Fig 5(b). The measured ratio response slopes are 
$0.027 \mathrm{~dB} /{ }^{0} \mathrm{C}, 0.042 \mathrm{~dB} /{ }^{0} \mathrm{C}$ and $0.068 \mathrm{~dB} /{ }^{0} \mathrm{C}$ respectively for $1530 \mathrm{~nm}, 1550 \mathrm{~nm}$ and 1570 $\mathrm{nm}$. This shows that for the fibre temperature sensor with a fixed bend radius, temperature sensitivity can be improved by changing the input signal wavelength. Given the resolution of the detection system, typically $0.01 \mathrm{~dB}$, a temperature sensor with bend radius of $11.5 \mathrm{~mm}$ with an input signal wavelength of $1530 \mathrm{~nm}$ can resolve $0.5^{0} \mathrm{C}$, while a higher temperature resolution of $0.25{ }^{\circ} \mathrm{C}$ and $0.1{ }^{\circ} \mathrm{C}$ can be achieved at $1550 \mathrm{~nm}$ and $1570 \mathrm{~nm}$ respectively.

To further analyze the system's temperature resolution, the temperature of the packaged sensor head is changed from $20{ }^{\circ} \mathrm{C}$ to $22{ }^{0} \mathrm{C}$ with a $0.5{ }^{0} \mathrm{C}$ step interval (limited by the capability of the temperature controller). The measured ratio variation versus temperature change at each wavelength is shown in Fig. 6. From the figure it is clear that a $0.5{ }^{0} \mathrm{C}$ temperature variation is clearly resolvable and the temperature resolution increases when a longer wavelength is used. For the system with an input signal wavelength of $1550 \mathrm{~nm}$ or $1570 \mathrm{~nm}$, a temperature resolution better than $0.5^{\circ} \mathrm{C}$ is clearly achievable.

\subsection{Effect of stress and strain on the temperature sensor accuracy}

It is well known that stress and strain applied to a bent fibre vary the bend loss and hence the ratio of the system will be affected and as a result errors will be induced in temperature measurements. Therefore it is necessary to carry out stress and strain study of the packaged fibre temperature sensor to estimate the stress and strain induced error in temperature measurements. To study the impact of stress on the sensor, the packaged sensor is placed between two solid metallic blocks to allow a stable application of a load as shown in Fig. 7(a). A load cell (SLB 250), which is placed in between the load applicator and the metallic block is used to measure the applied load. The output of the load cell is measured using a load cell data acquisition module SCC-SG24 combined with a data acquisition card PXI6221 from National Instruments. The system was programmed using LabVIEW 8.0 to obtain the applied load. The load was varied from 0 to $10 \mathrm{~N}$ with an interval of $1 \mathrm{~N}$ and the ratio of the system is recorded for each load at wavelengths $1530 \mathrm{~nm}, 1550 \mathrm{~nm}$ and $1570 \mathrm{~nm}$. The measured ratio variation for all the three wavelengths as load increases is shown in Fig. 8(a). The data were obtained at a room 
temperature of approximately $20{ }^{\circ} \mathrm{C}$. The ratio variation is linear in nature and for the sensor under test, the measured ratio variation was $0.042 \mathrm{~dB}, 0.068 \mathrm{~dB}$ and $0.071 \mathrm{~dB}$ respectively for wavelengths $1530 \mathrm{~nm}, 1550 \mathrm{~nm}$ and $1570 \mathrm{~nm}$ for an applied load of 10 N. Considering the temperature sensitivity of the sensor this gives rise to $0.15^{0} \mathrm{C} / \mathrm{N}, 0.18$ ${ }^{0} \mathrm{C} / \mathrm{N}$ and $0.16{ }^{\circ} \mathrm{C} / \mathrm{N}$ error in the temperature measurements respectively for input signal wavelengths of $1530 \mathrm{~nm}, 1550 \mathrm{~nm}$ and $1570 \mathrm{~nm}$. The estimated temperature error that could arise from the lateral load applied to the packaged sensor is shown in Fig. 8(b). From the figure it is clear that, the impact of load on the packaged sensor is not substantial, however since the ratio-stress response is linear, a correction can be applied to the temperature response and accurate temperature measurement is possible using the macro-bend temperature sensor for fixed load conditions.

For the packaged sensor, strain may originate due to bending of the packaged sensor or due to other deformations. It is very difficult to estimate the impact of local strain originating from the minor deformations, however due to the rigid and high compressive strength capability of the packaging material this phenomenon can be mitigated to some extent. In this study the focus is on a general estimation of strain influence when the packaged sensor experiences a uniform bending. To estimate the influence of strain on the temperature measurements of the packaged sensor, a $5 \mathrm{~mm}$ strain gauge is attached at the centre of the packaged sensor. One of the diagonally opposite ends of the packaged sensor with respect to the attached strain gauge is fixed to a solid surface and the load is applied to the other diagonally opposite end of the packaged sensor at a point $5 \mathrm{~mm}$ from the border as shown in Fig. 7(b). The strain at the centre of the sensor is measured using the attached strain gauge. It is assumed that the strain experienced at other areas of the packaged sensor is proportional to the strain at the centre of the sensor. However due to the minor differences in the thickness at different parts of the packaged sensor, there could be a small non-uniformity in the strain distribution. In this study our main focus is on the behavior of the ratio variation with strain, rather than an accurate strain mapping of the surface of the packaged sensor. To read the data from the strain gauge, a strain gauge module SCC-SG03 combined with a data acquisition card PXI6221 from National Instruments is used. The power ratio of the sensor system at wavelengths $1530 \mathrm{~nm}, 1550$ 
$\mathrm{nm}$ and $1570 \mathrm{~nm}$ is measured up to a strain level of $500 \mu \varepsilon$ at intervals of $50 \mu \varepsilon$. The measured ratio variation of the sensor system at all the three wavelengths due to the applied strain is shown in Fig. 9(a). From the figure it is clear that ratio variation shows a linear behavior with applied strain. The estimated temperature error due to the applied strain is presented in Fig. 9(b). The strain induced errors of the temperature sensing system with the present experimental condition are approximately $0.03{ }^{0} \mathrm{C} / 10 \mu \varepsilon, 0.03$ ${ }^{0} \mathrm{C} / 10 \mu \varepsilon$ and $0.02{ }^{0} \mathrm{C} / 10 \mu \varepsilon$ respectively for $1530 \mathrm{~nm}, 1550 \mathrm{~nm}$ and $1570 \mathrm{~nm}$. Thus from the studies on the impact of stress and strain on the packaged sensor, it is clear that the ratio shows a linear variation with stress and strain, and is not significantly high. If the fixed load and static strain is known, correction to the temperature response is possible due to the linear nature of the stress and strain response of the temperature sensor head.

\section{A portable prototype temperature sensor and interrogation system}

To demonstrate the simplicity of the sensor head and the interrogation system and also the ease of the development of the sensing interrogation system, a portable prototype of the temperature sensing system is constructed. The portable temperature sensing system is designed for a wavelength of operation at $1550 \mathrm{~nm}$ due to the availability of low cost single wavelength laser diodes at that wavelength. The laser used was a fibre pigtailed diode laser (LPS-1550-FC, Thorlabs) with a $1 \mathrm{~mW}$ maximum output power, which is driven by a constant power laser driver current unit (LD100, Thorlabs). The system is implemented as a ratiometric scheme, where the input power from the laser is split into two using a $3 \mathrm{~dB}$ coupler, one part of the signal is directed to the photo detector through the temperature sensor, while the other acts as a reference arm, which is connected directly to a second photo detector. The photo detectors used were InGaAs fibre optic photo detectors (D400FC) from Thorlabs. The outputs from the photo detectors are connected to a USB data acquisition card (USB 6009) from National Instruments. The whole system is connected to a laptop computer via a USB cable and is interfaced using LabVIEW Software from National Instruments. The sensor is calibrated for a temperature range of $0-80{ }^{0} \mathrm{C}$ and the calibration data is fed into the control program, which is then used to compare the measured data to obtain the real time temperature information. The 
developed prototype of the portable sensing system is shown in Fig. 10. Thus in this section we have shown that a macro-bend fibre based temperature sensors at its ratiometric intensity based interrogation system can be built with readily available low cost components and hence it is a viable and low cost solution that can be adapted for many engineering applications where point temperature measurements are required.

\section{Conclusion}

An epoxy packaged surface mountable fibre temperature sensor for engineering applications is demonstrated in this paper. From the studies on the influence of the absorption coating on the linearity of the temperature response of the sensor it is concluded that a complete absorption of radiation modes is necessary for a macro-bend fibre to be used as a temperature sensor. The temperature response of the sensor head is tested up to $80{ }^{\circ} \mathrm{C}$. It is shown that, for a fixed wavelength, the temperature sensitivity can be improved by reducing the bend radius. To allow the sensor to be used for engineering applications, the sensor head is packaged in a high compressive strength and low thermal expansion coefficient two-part epoxy. The temperature response and resolution of the packaged system is tested and it is demonstrated that the sensor gives a linear temperature response with a resolution better than $0.5{ }^{0} \mathrm{C}$. For a fixed bend radius fibre temperature sensor, the temperature resolution can be improved by using a longer operating wavelength. For an input signal wavelength of $1570 \mathrm{~nm}$, a temperature resolution of $0.1{ }^{0} \mathrm{C}$ is achievable. The stress and strain behaviors of the packaged sensor are studied and it is found that, the responses are approximately linear. The stress and strain induced errors in the sensing system with the experimental condition presented in this study are $0.15{ }^{0} \mathrm{C} / \mathrm{N}, 0.18{ }^{0} \mathrm{C} / \mathrm{N}$ and $0.16{ }^{\circ} \mathrm{C} / \mathrm{N}$ and $0.03{ }^{0} \mathrm{C} / 10 \mu \varepsilon, 0.03{ }^{0} \mathrm{C} / 10 \mu \varepsilon a n d$ $0.02{ }^{0} \mathrm{C} / 10 \mu \varepsilon$ respectively for $1530 \mathrm{~nm}, 1550 \mathrm{~nm}$ and $1570 \mathrm{~nm}$. Due to linear nature of the stress and strain response, a correction can be applied to the temperature response and accurate temperature measurement is possible using the macro-bent temperature sensor under a fixed load and strain. A portable prototype of the sensor head and the interrogation system is also built and demonstrated. The demonstrated temperature sensing system could be used for a wide range of engineering applications. 


\section{References}

[1] L. Faustini and G. Martini, Bend loss in singlemode fibers J. Lightwave Technol. 5 (1992) 671-679.

[2] H. Renner, Bend losses of coated singlemode fibers: A simple approach, J. Lightwave Technol. 10 (1992) 544-551.

[3] Q. Wang, G. Farrell, T. Freir, G. Rajan and P. Wang, Low-cost wavelength measurement based on a macrobending single-mode fiber, Opt. Lett. 31 (2006) 17851787.

[4] F. M. Haran, J. S Barton, S. R. Kidd and J. D. C Jones, Optical fibre interferometric sensors using buffer guided light, Meas. Sci. Technol. 5 (1991) 526-530.

[5] S. H. Nam, and S. Yin, High temperature sensing using whispering gallery mode resonance in bent optical fibers, IEEE Photonics. Technol. Lett. 17 (2005) 2391-2393.

[6] Byoungho Lee, Review of present status of optical fiber sensors, Optical fiber technology. 9 (2003) 57-79.

[7] K. O. Hill, G Meltz, Fiber Bragg grating technology fundamentals and overview, J. Lightwave Technol. 15 (1997) 1263 - 1276.

[8] Y. Zhao and Y. Liao, Discrimination methods and demodulation techniques for fiber Bragg grating sensors, Optics and Lasers In Engineering. 41( 2005) 1-18

[9] G. Rajan, Y. Semenova and G. Farrell, All-fibre temperature sensor based on macrobend singlemode fibre loop, Electron. Lett. 44 (2008)1123-1124.

[10] D. Marcuse, Curvature loss formula for optical fibers, J. Opt. Soc. Am. 66 (1976) 216-220.

[11] P. Wang, G. Rajan, Y. Semenova and G. Farrell, Temperature dependence of a macrobending edge filter based on a high-bend loss fiber, Opt. Lett. 33 (2008) 24702472.

[12] P. Wang, G. Farrell, Q. Wang and G. Rajan, An optimized macro-bending fiber based edge filter, IEEE Photonics. Technol. Lett. 19 (2007) 1136-1138 


\section{Figure Captions}

Figure 1. (a) A macro-bend fibre temperature sensor head (b) Schematic of the temperature sensor system

Figure 2. Transmission response of the different coating materials from 400-1700 nm (a) $0-100 \%$ (b) $0-5 \%$

Figure 3. (a) Comparison of temperature response of the macro-bend fibre with different absorption coatings (b) Temperature response of the sensor coated with Indian ink for two different bend radii

Figure 4. Packaging step sequence for the sensor (a) Aluminium tray with the sensor fixed (b) Epoxy filled tray (c) packaged fibre temperature sensor

Figure 5. Ratio response of the sensor before and after packaging (a) versus wavelength (b) versus temperature

Figure 6. Plots showing temperature resolution. Ratio variation of the sensor with a step change on $0.5{ }^{\circ} \mathrm{C}$ from $20{ }^{\circ} \mathrm{C}$ to $22{ }^{\circ} \mathrm{C}$ (a) at $1530 \mathrm{~nm}$ (b) at $1550 \mathrm{~nm}$ (c) at $1570 \mathrm{~nm}$

Figure 7. (a) Experimental arrangement to study the stress impact on the sensor (b) Experimental arrangement to study strain impact on the sensor

Figure 8. (a) Stress induced ratio variation of the packaged temperature sensor (b) Estimated stress induced error in temperature measurements

Figure 9. (a) Strain induced ratio variation of the packaged temperature sensor (b) Estimated strain induced error in temperature measurements

Figure 10. Photograph of the portable prototype of the temperature sensor interrogation system 


\section{Figures}

Figure 1

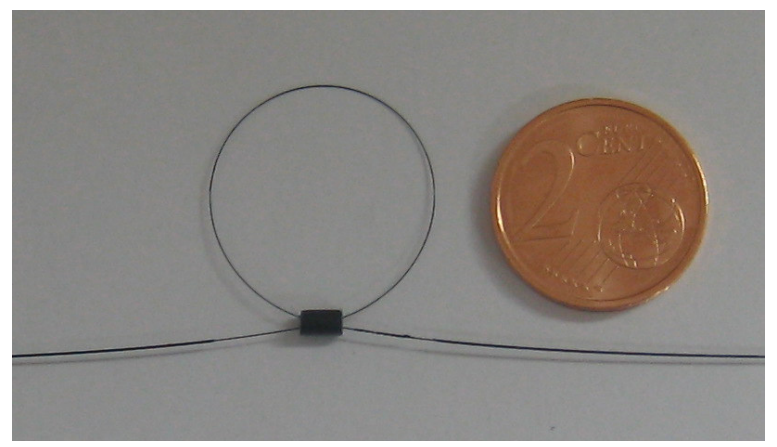

(a)

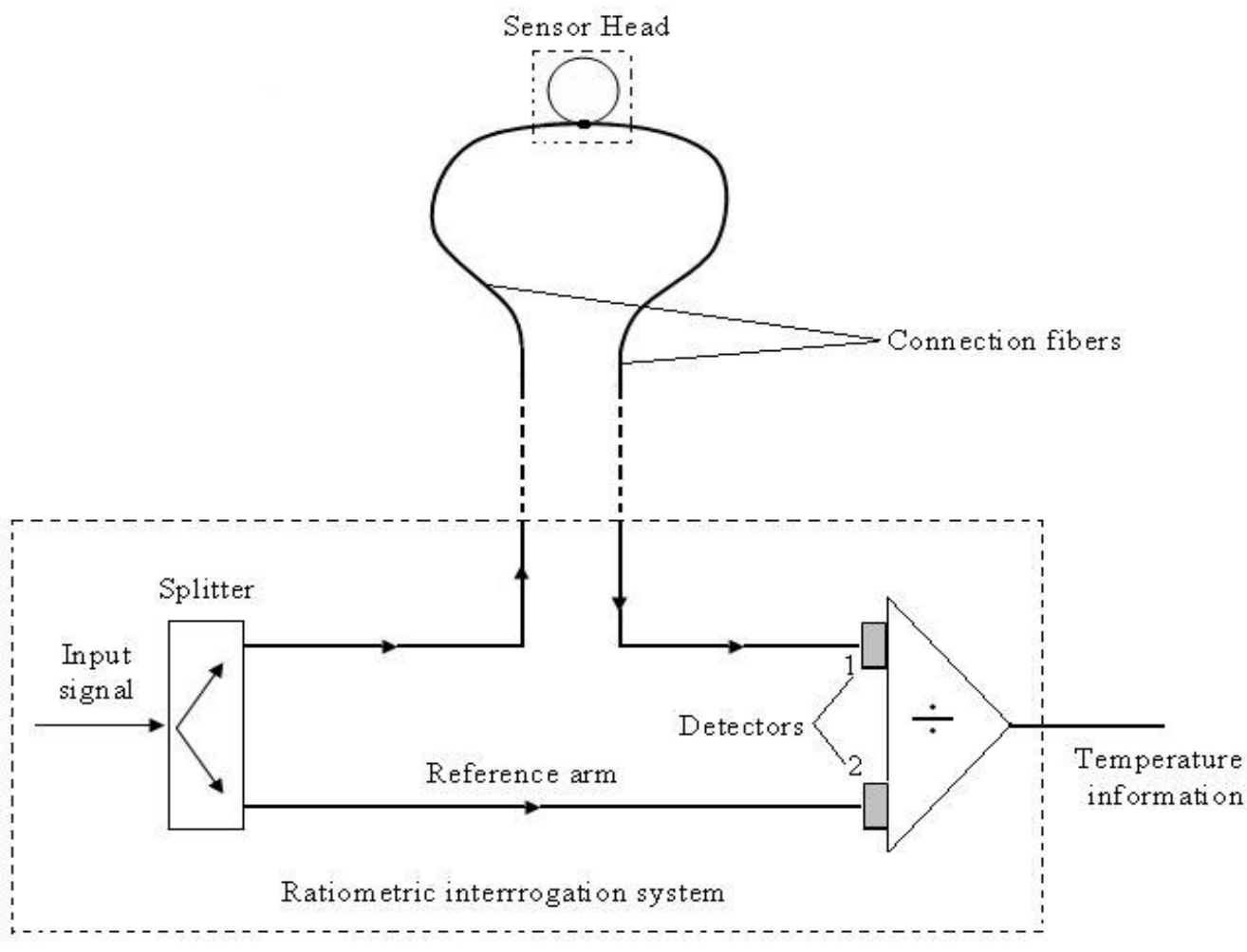

(b) 
Figure 2

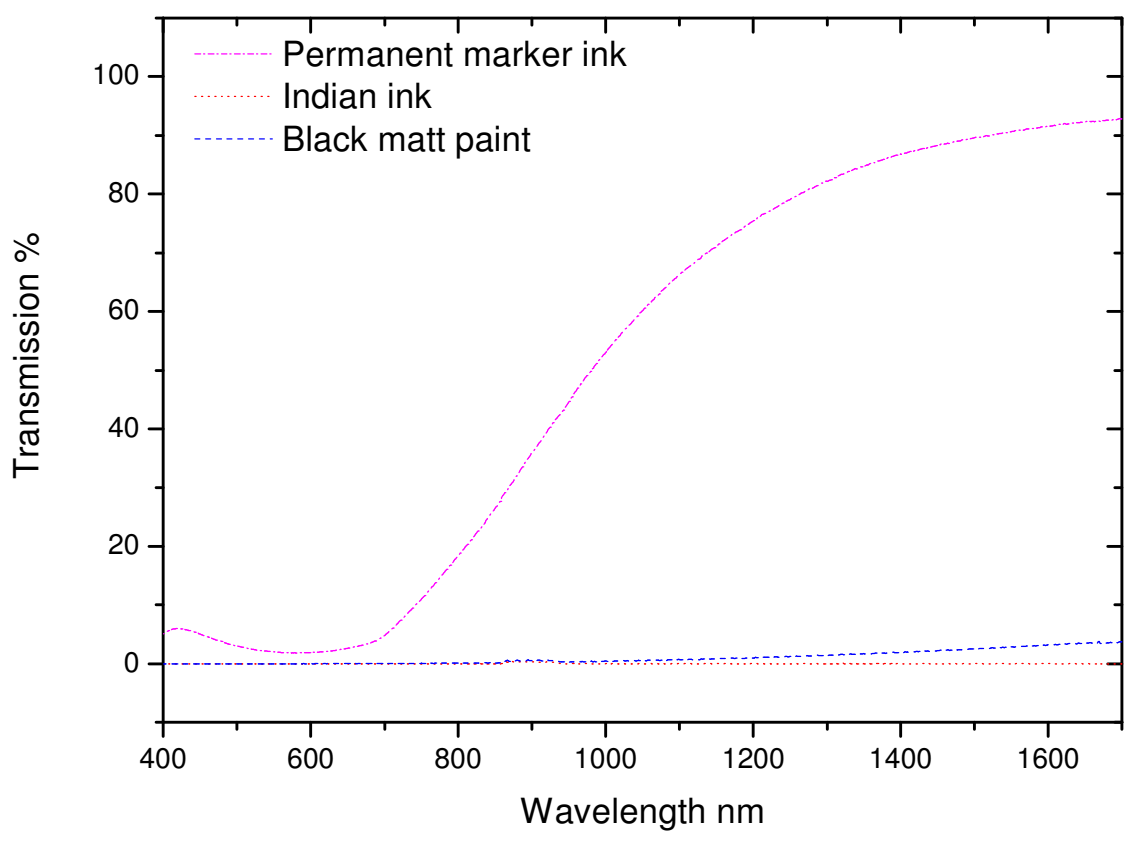

(a)

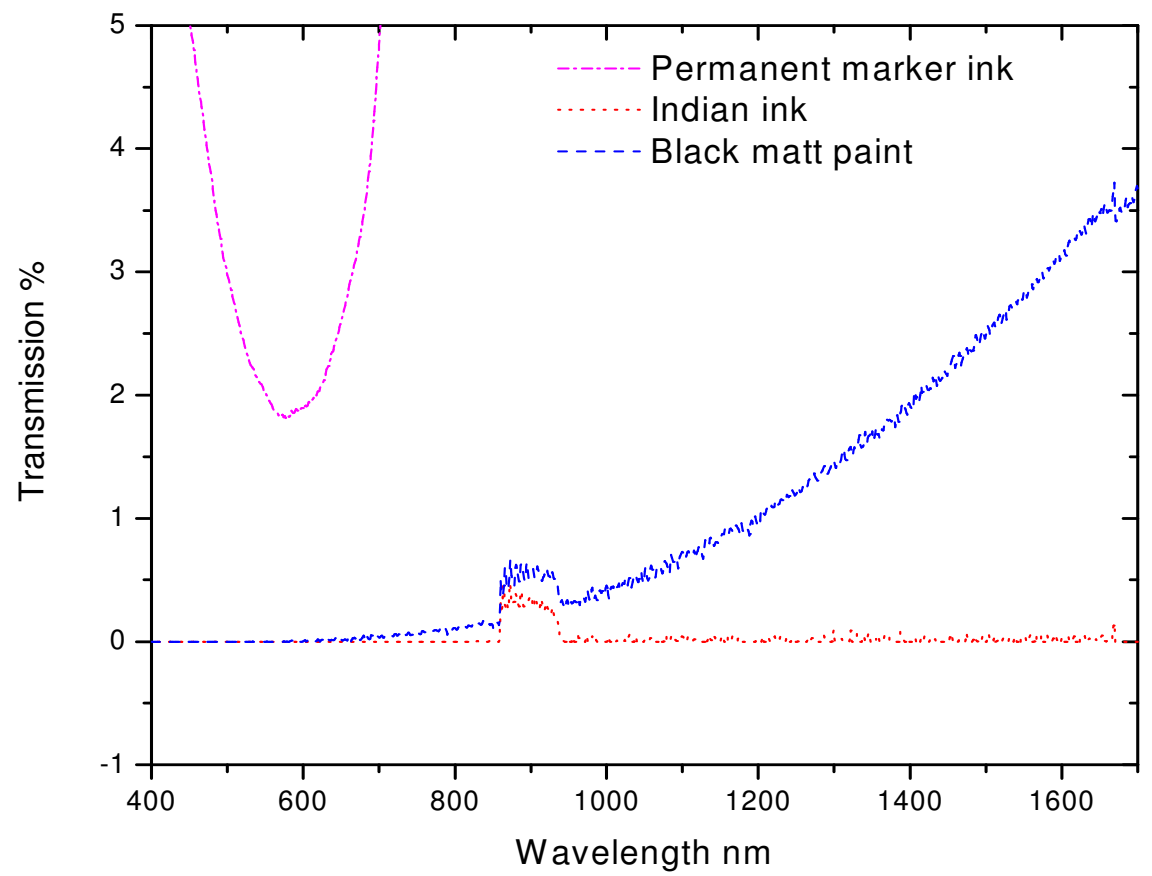

(b) 
Figure 3

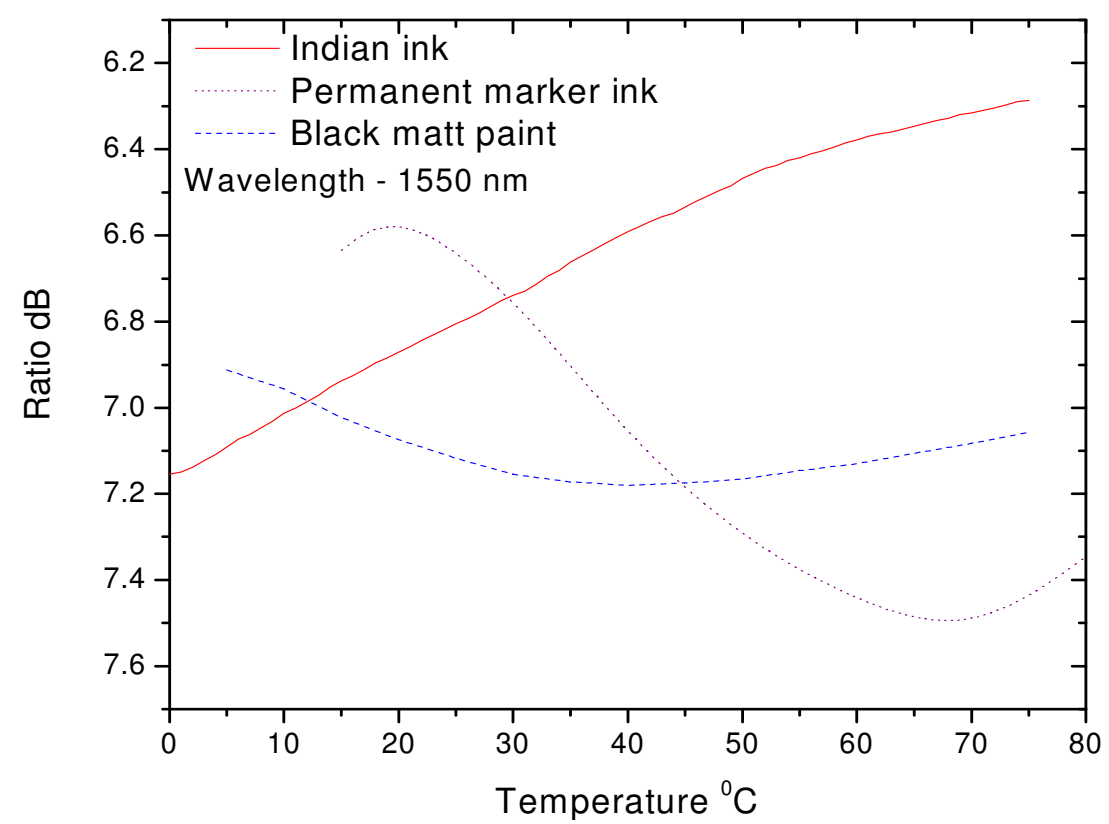

(a)

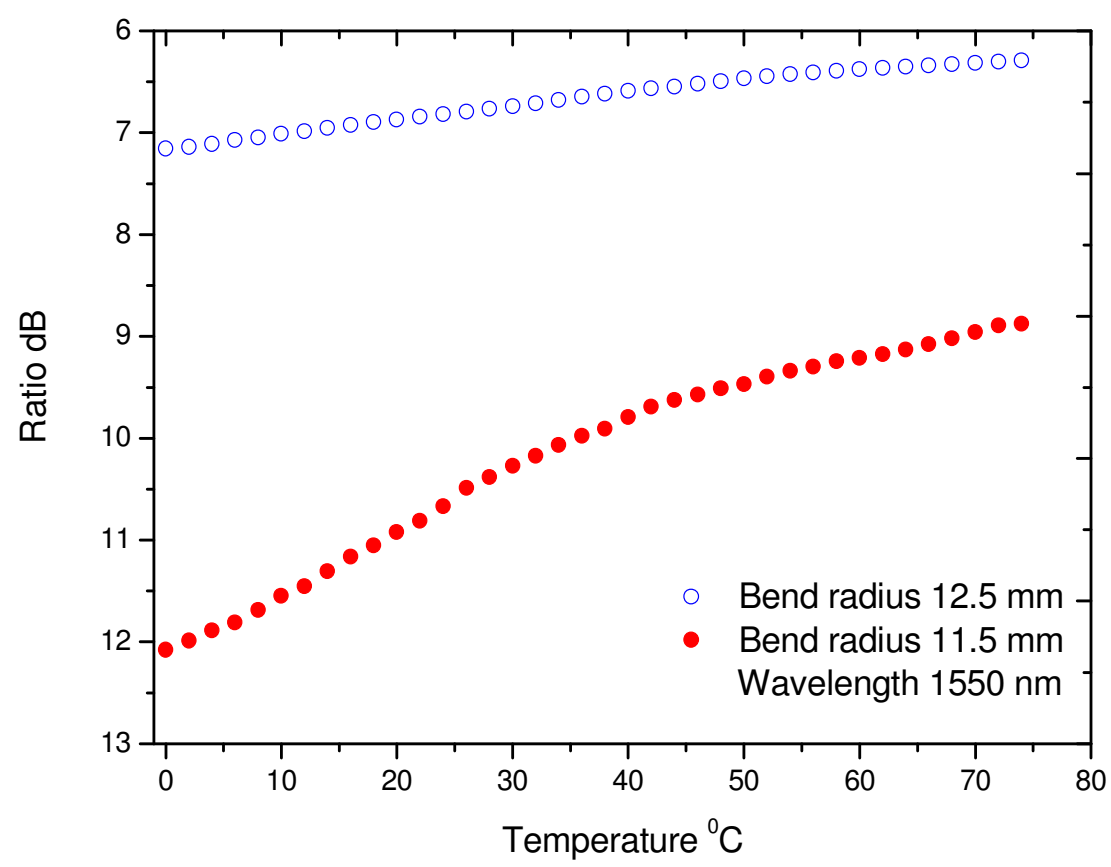

(b) 
Figure 4

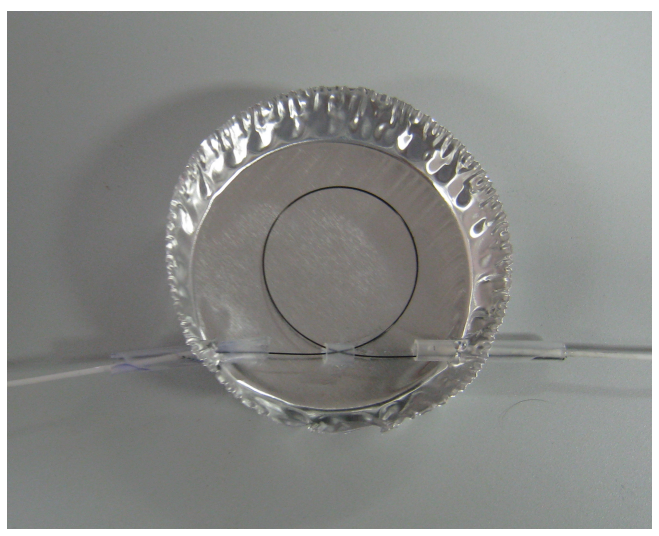

(a)

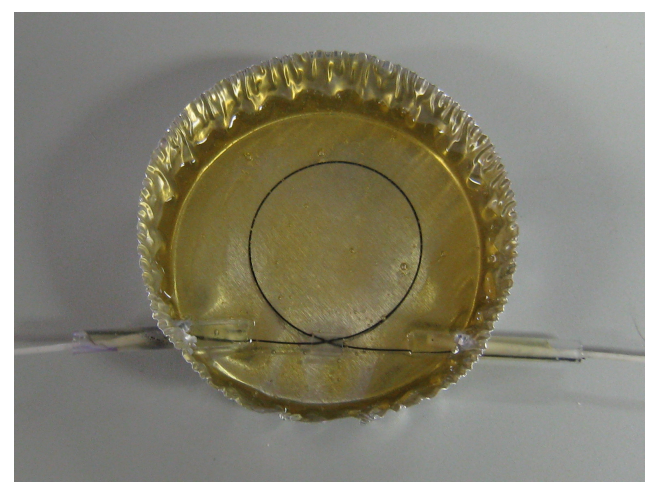

(b)

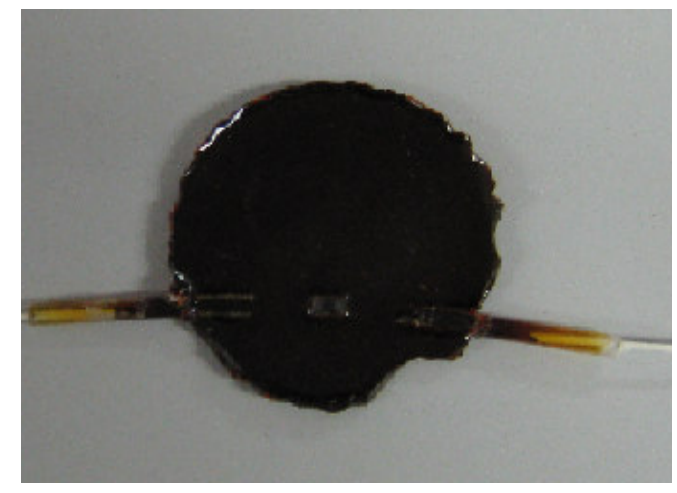

(c) 


\section{Figure 5}

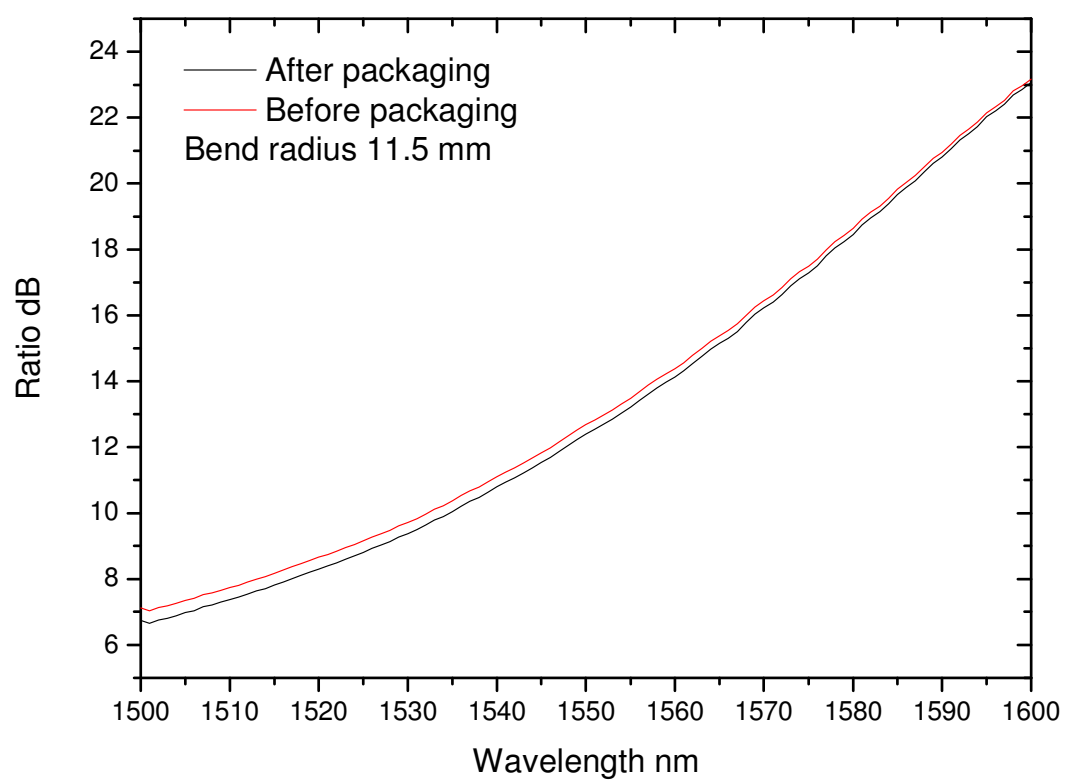

(a)

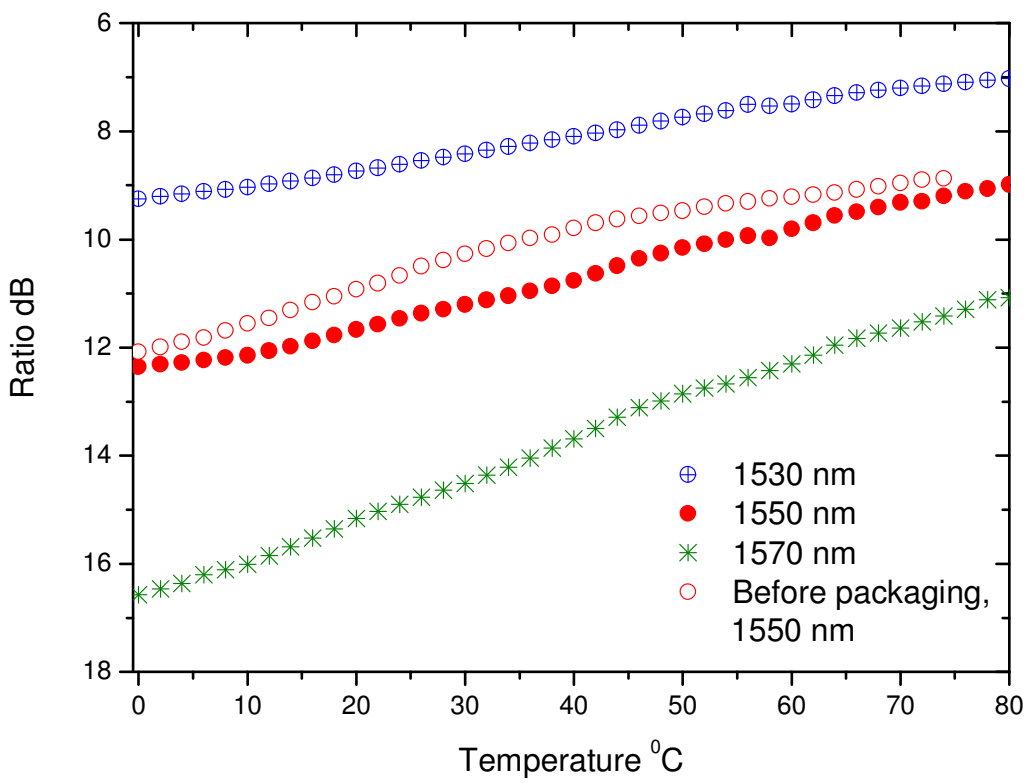

(b) 
Figure 6

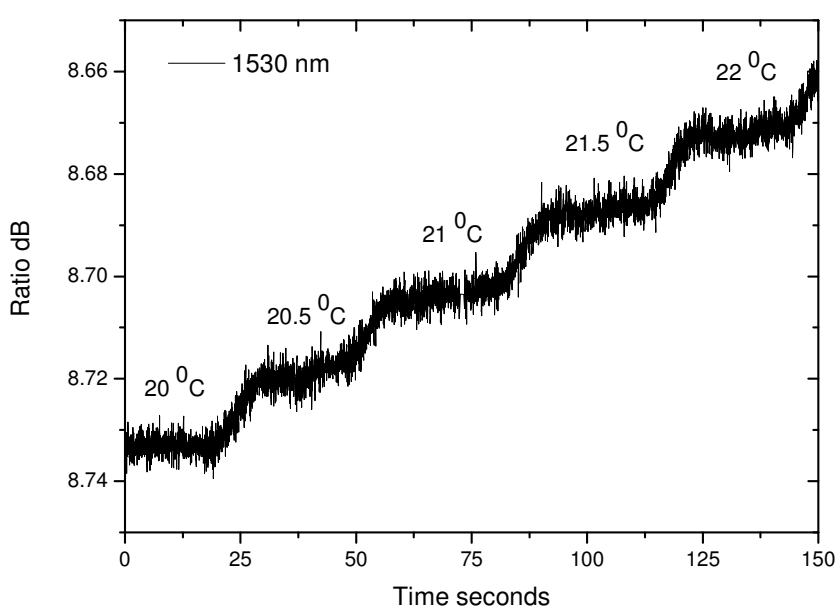

(a)

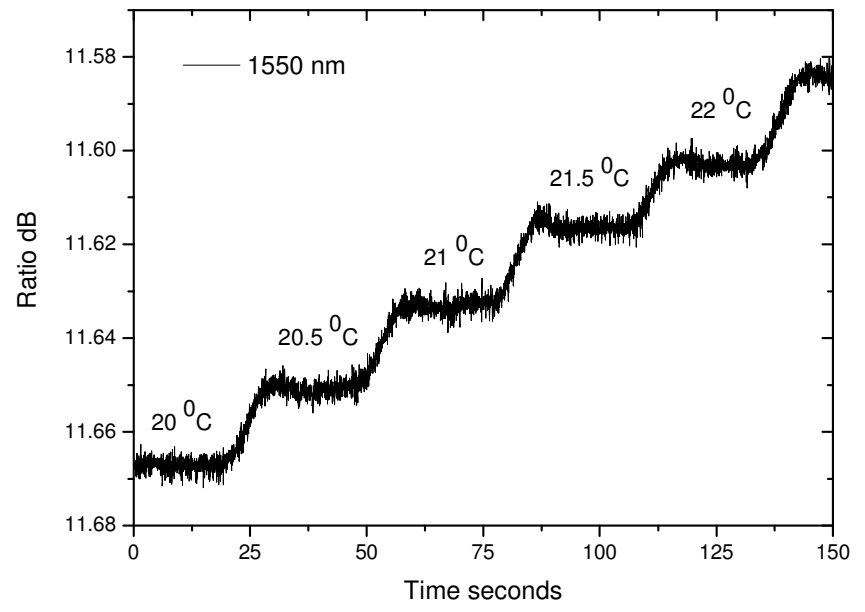

(b) 


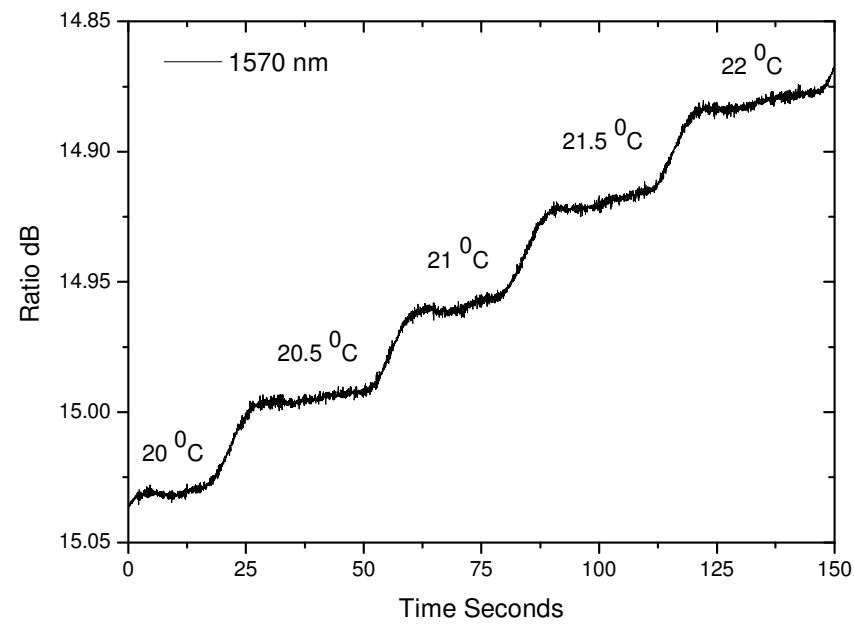

(c)

Figure 7

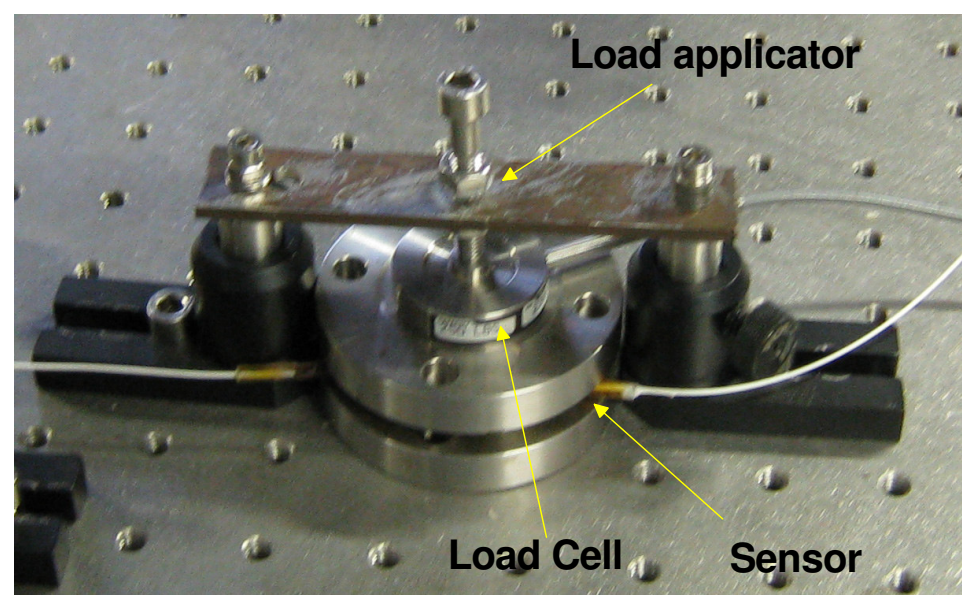

(a) 


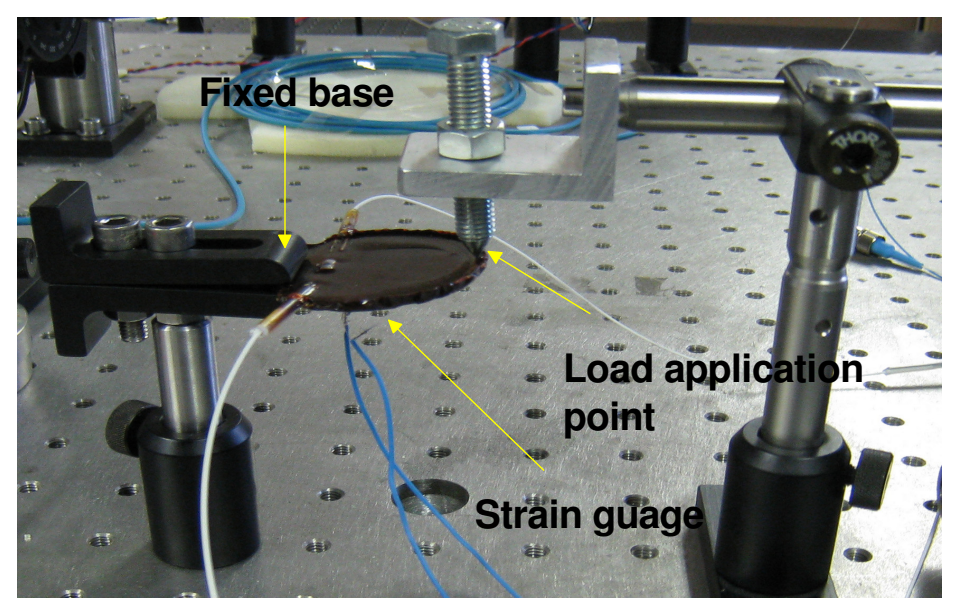

(b)

Figure 8

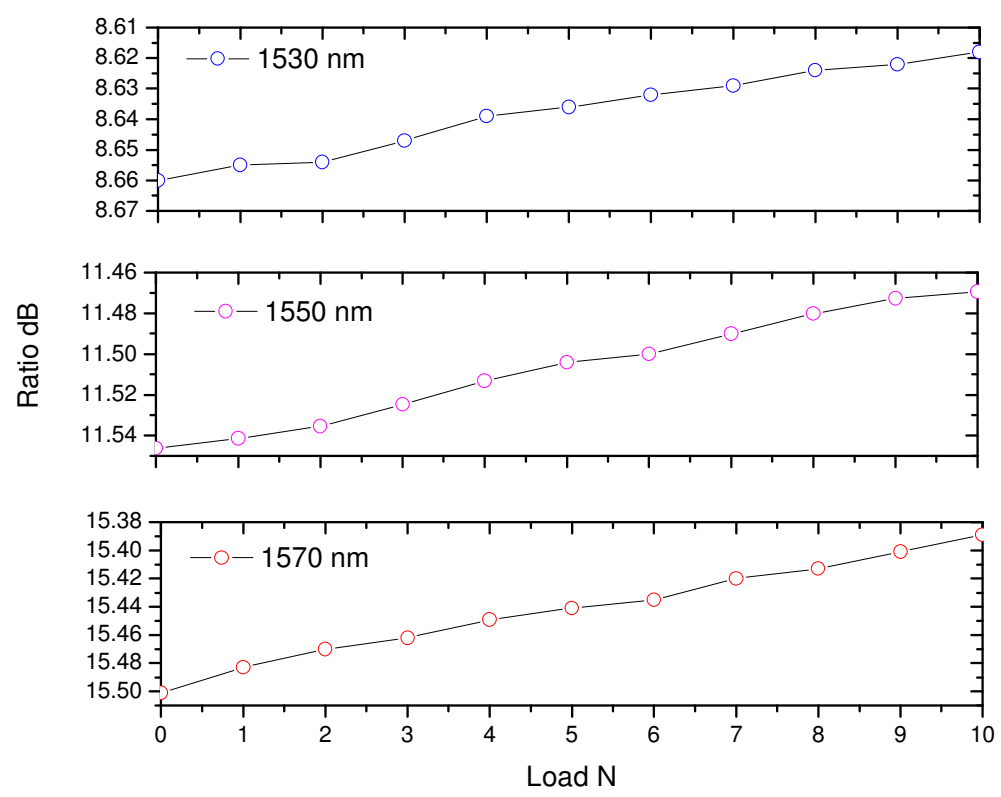

(a) 


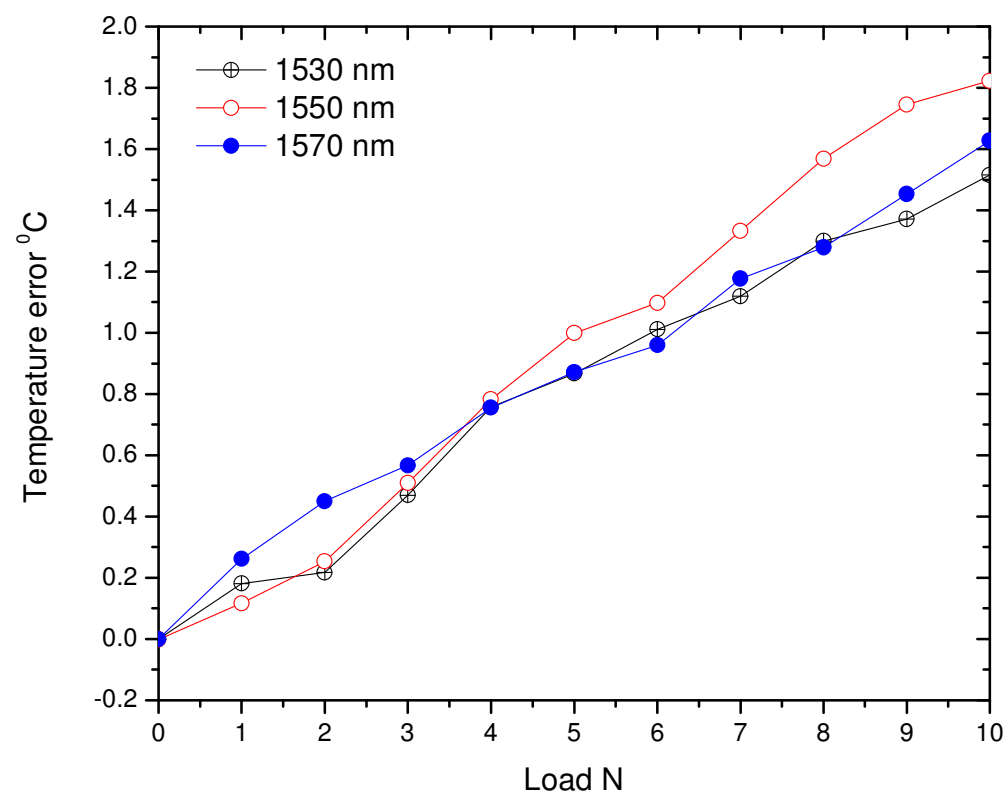

(b)

Figure 9

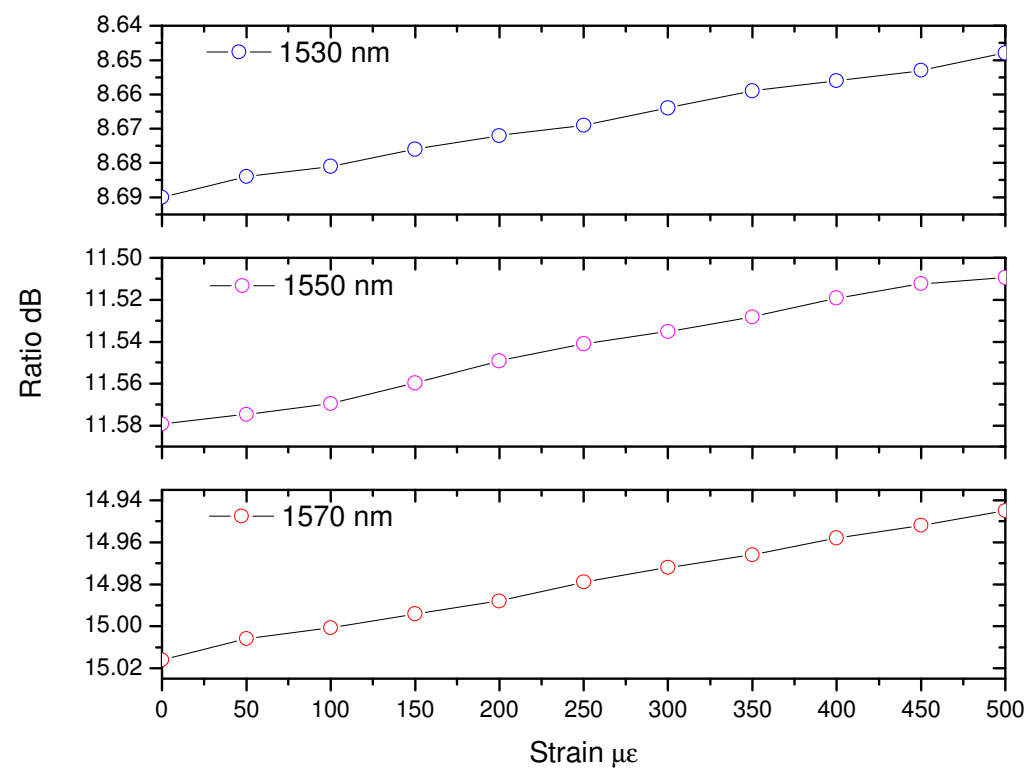

(a) 


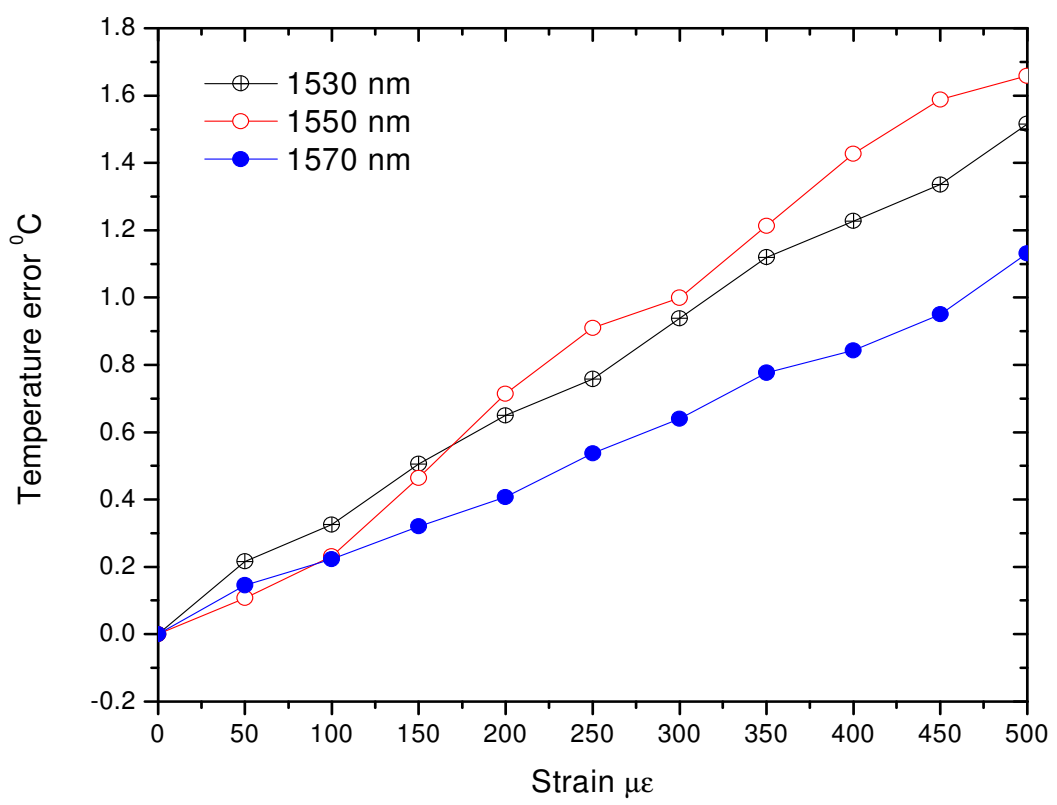

(b)

Figure 10

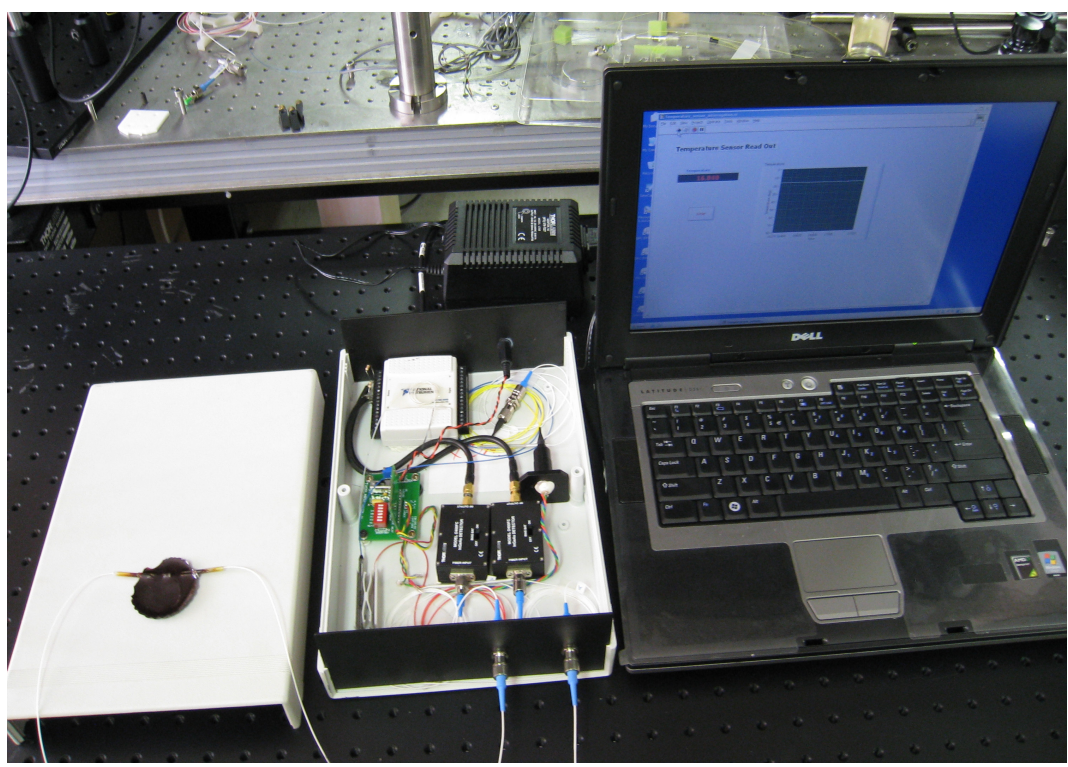

